\title{
BMJ Open Symptoms of common mental disorders and adherence to antiretroviral therapy among adults living with HIV in rural Zimbabwe: a cross-sectional study
}

To cite: Haas AD,

Kunzekwenyika C, Hossmann S, et al. Symptoms of common mental disorders and adherence to antiretroviral therapy among adults living with HIV in rural Zimbabwe: a crosssectional study. BMJ Open 2021;11:e049824. doi:10.1136/ bmjopen-2021-049824

- Prepublication history for this paper is available online. To view these files, please visit the journal online (http://dx.doi. org/10.1136/bmjopen-2021049824).

Received 03 February 2021 Accepted 18 June 2021

Check for updates

(C) Author(s) (or their employer(s)) 2021. Re-use permitted under CC BY-NC. No commercial re-use. See rights and permissions. Published by BMJ.

For numbered affiliations see end of article.

Correspondence to Dr Andreas D Haas; andreas.haas@ispm.unibe.ch

\section{ABSTRACT}

Objectives To examine the proportion of people living with HIV who screen positive for common mental disorders (CMD) and the associations between CMD and selfreported adherence to antiretroviral therapy (ART).

Setting Sixteen government-funded health facilities in the rural Bikita district of Zimbabwe.

Design Cross-sectional study.

Participants HIV-positive non-pregnant adults, aged 18 years or older, who lived in Bikita district and had received ART for at least 6 months.

Outcome measures The primary outcome was the proportion of participants screening positive for CMD defined as a Shona Symptoms Questionnaire score of 9 or greater. Secondary outcomes were the proportion of participants reporting suicidal ideation, perceptual symptoms and suboptimal ART adherence and adjusted prevalence ratios (aPR) for factors associated with CMD, suicidal ideation, perceptual symptoms and suboptimal ART adherence.

Results Out of 3480 adults, $18.8 \%$ (95\% Cl $14.8 \%$ to $23.7 \%$ ) screened positive for CMD, $2.7 \%$ (95\% Cl 1.5\% to $4.7 \%)$ reported suicidal ideations, and $1.5 \%(95 \% \mathrm{Cl}$ $0.9 \%$ to $2.6 \%$ ) reported perceptual symptoms. Positive CMD screens were more common in women (aPR 1.67, $95 \% \mathrm{Cl} 1.19$ to 2.35 ) than in men and were more common in adults aged $40-49$ years (aPR $1.47,95 \% \mathrm{Cl} 1.16$ to 1.85 ) or aged $50-59$ years (aPR $1.51,95 \% \mathrm{Cl} 1.05$ to 2.17 ) than in those 60 years or older. Positive CMD screen was associated with suboptimal adherence (aPR 1.53; $95 \% \mathrm{Cl}$ 1.37 to 1.70 ).

Conclusions A substantial proportion of people living with HIV in rural Zimbabwe are affected by CMD. There is a need to integrate mental health services and HIV programmes in rural Zimbabwe.

Trial registration number NCT03704805.

\section{BACKGROUND}

In 2019, approximately 1.4 million people were living with HIV in Zimbabwe, of whom more than 1.1 million were receiving antiretroviral therapy (ART). ${ }^{1}$ Widespread access to
Strengths and limitations of this study

- Inclusion of a large sample of people living with HIV recruited at 16 government-funded primary and secondary care facilities in a rural district of Zimbabwe.

- Use of a locally developed screening tool that showed good psychometric properties for detecting common mental disorders in Zimbabwe in HIV-positive urban populations.

- The screening tool was not validated for the rural setting, and the cut-off score was selected based on data from the urban setting.

Adherence to antiretroviral therapy was self-reported.

ART has dramatically improved the life expectancy of people living with HIV. ${ }^{2}$ However, the long-term effectiveness of ART depends on lifelong retention in HIV care and strict medication adherence. ${ }^{34}$

Common mental disorders (CMD), which include depression and anxiety disorders are highly prevalent among people living with HIV. In sub-Saharan Africa, the estimated prevalence of major depression in people living with HIV is $15.3 \%$, and of depressive symptoms $27.0 \%{ }^{5}$ The median prevalence of anxiety disorders in people living with HIV in low/middle-income countries is estimated at $22.8 \%{ }^{6}$ The prevalence of depression and anxiety disorders is higher in women than in men. ${ }^{78}$ In Zimbabwe's capital Harare, over $50 \%$ of people living with HIV attending a primary care facility met diagnostic criteria for either depression or anxiety, and $65 \%$ screened positive for CMD. ${ }^{9}{ }^{10}$ In Zimbabwe, most people living with HIV receiving ART reside in rural areas, ${ }^{11}$ and the CMD prevalence in this population is unknown. 
The co-occurrence of mental disorders and HIV poses significant challenges in managing and treating HIV. Mental disorders are associated with poor HIV treatment outcomes, including low adherence, lack of viral load suppression, loss to follow-up and mortality. ${ }^{12-16}$ Early detection and effective management of mental disorders among people living with HIV may improve the quality of life of affected individuals, ART adherence and viral load suppression, ${ }^{1718}$ thus reducing the incidence of HIVassociated complications, preventing drug resistance and HIV transmission. Despite these benefits, there is a large 'treatment gap' in mental healthcare among people living with HIV in low-income and middle-income countries: most people affected by mental illness do not receive appropriate treatment. ${ }^{1920}$

The Friendship Bench intervention is a culturally adapted evidence-based psychological intervention developed to close the treatment gap for CMD in Zimbabwe. ${ }^{21}$ The Friendship Bench team trains community health workers to identify people with CMD symptoms and deliver a brief intervention consisting of six sessions of problem-solving therapy and optional group support. ${ }^{21}$ We are conducting a cluster randomised trial to assess the effectiveness of the Friendship Bench intervention in improving ART outcomes and symptoms of CMD in people living with HIV in rural Zimbabwe. During recruitment, we screened over 3500 ART patients for CMD and poor adherence. In this paper, we report the prevalence of positive CMD screening tests and associations between positive CMD screens and self-reported adherence among people living with HIV in rural Zimbabwe.

\section{METHODS}

We conducted a cross-sectional study at 16 health facilities in Bikita district to assess the eligibility of individuals for a cluster randomised trial (FB-ART) on the effect of the Friendship Bench intervention ${ }^{22}$ on ART outcomes and symptoms of CMD in people living with HIV. Bikita is a rural district of the Masvingo Province about $300 \mathrm{~km}$ south of Harare.

Between 5 October 2018 and 19 December 2019, we offered CMD screening at 16 government-funded health facilities in rural Zimbabwe. HIV-positive non-pregnant adults aged 18 years or older who lived in Bikita district and had received ART for at least 6 months were eligible. Trained research assistants offered screening for CMD using the Shona Symptoms Questionnaire (SSQ-14) ${ }^{9}$ and assessed adherence using a question from the AIDS indicator survey. ${ }^{23}$ The SSQ-14 is a locally developed CMD screening tool. ${ }^{9}$ The tool assesses if individuals had experienced common mental health symptoms, including sleep disturbance, suicidal ideations, tearfulness, perceptual symptoms and impairment of functioning in the past 7 days. Each of the 14 symptoms is scored dichotomously (symptom present or absent). ${ }^{24}$ The tool showed good psychometric properties for detecting CMD in HIV-positive and HIV-negative urban populations in
Zimbabwe. ${ }^{9}$ An SSQ-14 score of $\geq 9$ had a sensitivity of $88 \%$ and a specificity of $76 \%$ for depression or general anxiety in HIV-positive adults in Harare. ${ }^{9}$ The tool had a high internal consistency in the validation study (Cronbach's $\alpha=0.74$ ) and in our study (Cronbach's $\alpha=0.82$ ). Adherence was assessed based on self-report using the following question: 'In the last 30 days, how many days have you missed taking any of your ARV [antiretroviral] pills?'. 23

We defined SSQ-14 scores of 9 or greater as positive CMD screen. ${ }^{9}$ Participants who reported that they 'felt like committing suicide' in the past 7 days (SSQ-14 item 11) screened positive for suicidal ideation. Participants who reported that they 'saw or heard things which others could not see or hear' in the past 7 days (SSQ-14 item 5) screened positive for perceptual symptoms. Participants who indicated that they had missed taking one or more ARV pills in the last 30 days were classified as having suboptimal adherence. Those reporting not having missed any ARV pills had optimal adherence. We categorised age into the following groups: 18-29 years, 30-39 years, $40-49$ years, $50-59$ years and 60 years or older.

Individuals who participated in SSQ screening were eligible for this analysis. Participants who did not respond to all $14 \mathrm{SSQ}$ items were excluded. We calculated the prevalence of non-adherence and positive screening outcomes with logit-transformed 95\% CIs that adjusted for intragroup correlation at health facilities. We estimated adjusted prevalence ratios (aPRs) for factors associated with positive screening for CMD, suicidal ideation and perceptual symptoms using mixed-effects Poisson regression models with robust standard errors. ${ }^{25}$ Models were adjusted for sex, age and clustering of data at facilitylevel using a random intercept for study facilities. We used the same models to calculate unadjusted and aPRs for factors associated with suboptimal adherence. Statistical analysis was done using Stata (V.16, Stata).

Individuals who screened positive for CMD and provided written informed consent were included in the trial and were offered CMD treatment as part of the FB-ART (friendship bench-antiretroviral therapy) trial.

\section{Patient and public involvement}

Patients with psychiatric morbidity were involved in ethnographic and qualitative research, which informed the development of the SSQ-14. ${ }^{24}$ The results of this study were shared with the provincial medical director and the district medical officer.

\section{RESULTS}

Research assistants assessed the eligibility for CMD screening of 3707 individuals: $3543(95.6 \%)$ of eligible individuals participated in CMD screens. Pregnancy, residency outside of Bikita district and age below 18 years were the most common reasons for ineligibility for screening. Out of 3543 individuals screened for CMD, $63(1.7 \%)$ did not respond to all SSQ-14 items and were 
excluded. The remaining 3480 individuals were included in the analysis. The median age of the study population was 45 years (IQR 38-53). Three-quarters of the participants $(74.9 \%, \mathrm{n}=2608)$ were women.

Table 1 shows the prevalence and aPRs for positive screening for CMD, suicidal ideation and perceptual symptoms. Out of 3480 adults, $18.8 \%$ (95\% CI $14.8 \%$ to $23.7 \%, \mathrm{n}=655)$ screened positive for CMD, 2.7\% (95\% CI $1.5 \%$ to $4.7 \%, \mathrm{n}=93$ ) reported suicidal ideation, and $1.5 \%$ (95\% CI $0.9 \%$ to $2.6 \%, \mathrm{n}=52$ ) reported perceptual symptoms. Positive CMD screens were more common in women $(21.0 \%$; aPR $1.67,95 \%$ CI 1.19 to 2.35$)$ than in men $(12.4 \%)$ and were more common in adults aged $40-49$ years (20.6\%; aPR $1.4795 \%$ CI 1.16 to 1.85 ) or aged $50-59$ years (20.3\%; aPR $1.5195 \%$ CI 1.05 to 2.17 ) than in those 60 years or older $(12.6 \%)$. Suicidal ideations were more common in adults $18-29$ years $(4.7 \%, 95 \%$ CI $0.2 \%$ to $9.2 \%)$ and in adults aged $30-39$ years $(4.0 \%, 95 \% \mathrm{CI}$ $1.1 \%$ to $7.0 \%$ ) than in older adults, but the statistical uncertainty around these estimates was large.

Out of 3469 individuals who responded to the adherence question (11, $0.32 \%$ did not respond), 2900 (83.6\%, $95 \%$ CI $80.0 \%$ to $87.2 \%$ ) reported optimal adherence and 569 (16.4\%, 95\% CI 12.8\% to 20.0\%) reported suboptimal adherence. Suboptimal adherence was more common in individuals screening positive for CMD (aPR 1.53, 95\% CI 1.37 to 1.70 ) than in those screening negative (table 2). Suboptimal adherence was also more common in men (aPR 1.25, 95\% CI 1.01 to 1.53) than in women and adults aged $18-29$ years (aPR 1.62, 95\% CI 1.10 to 2.38 ) than in those 60 years or older (table 2 ).

\section{DISCUSSION}

In a rural district of Zimbabwe, about one in five HIV positive adult screened positive for CMD, almost $3 \%$ reported suicidal ideation, and $1.5 \%$ perceptual symptoms compatible with psychosis. Positive CMD screens were more common in women and middle-aged adults than in men or older adults. Positive CMD screens were associated with suboptimal self-reported ART adherence.

Our results have to be considered in light of two limitations. First, we used a locally developed CMD screening tool that had been validated in urban Zimbabwe, but we did not validate the tool for the rural setting and selected the CMD threshold based on the urban validation study. ${ }^{9}$ Second, we used a self-reported adherence measure which might be prone to under-reporting of non-adherence. However, there is evidence for the validity of self-reported measures of ART adherence. ${ }^{26}$ The strengths of our study include a large sample size and a multicentre design.

In line with the national ${ }^{10}$ and international literature, ${ }^{27} 28$ we found that CMDs were much more common in women than in men. Biological factors, including sex hormones and sex differences in the neuroendocrine response to stress, psychosocial factors such as gender differences in interpersonal orientation, self-esteem, body shaming and rumination might contribute to the gender gap in CMD. ${ }^{78}$ In addition to these individual-level factors, gender inequity and higher exposure of women and girls to traumatising life events, including genderbased violence or sexual abuse, may further contribute to the gender gap in CMD. ${ }^{71029}$

The prevalence of positive CMD screens observed in our study of a rural HIV positive population was less than one-third of the prevalence observed in a study of a similar urban population conducted in Harare in 2013. ${ }^{9}{ }^{10}$ An important factor that likely contributed to the lower prevalence of CMD in our study compared with earlier studies is the change of national ART guidelines to treat people living with HIV at less advanced stages of HIV disease. In the last decade, the CD4 threshold for ART eligibility was successively raised from $<250$ cells/ $\mu \mathrm{L}$ to immediate ART initiation of all people living with HIV under WHO's 'treat all' guidelines. These changes were reflected in an increase in the median $\mathrm{CD} 4$ at ART initiation in low and middle income. ${ }^{30} 31$ Low CD4 cell count is associated with a higher risk of $\mathrm{CMD},{ }^{32}$ and the comparably low prevalence of CMD observed in our study might reflect the higher median CD4 cell count at ART initiation. The gap in CMD prevalence between the urban and the rural setting might also be explained by a high prevalence of adverse living condition in the urban areas: $42 \%$ of the HIV positive individuals included in the urban study were unemployed, and $92 \%$ reported that they experienced a negative life event (eg, death in the family, physical or sexual assault, forced eviction, HIV diagnosis or hospitalisation of the participant or an immediate family member) in the 6 months before data collection. ${ }^{910}$ Furthermore, cultural differences in symptoms presentation between urban and rural populations might explain differences in the prevalence between the two settings. ${ }^{33}$

Our results confirm earlier data on associations between symptoms of depression and anxiety disorders and suboptimal adherence to ART. ${ }^{12}{ }^{15}$ A meta-analysis of eight studies from low-income countries found that the odds of suboptimal adherence were $92 \%$ higher in patients with depressive symptoms than those without depressive symptoms (OR 1.92, 95\% CI 1.47 to 2.5 ). ${ }^{15}$ Another meta-analysis of 11 studies from low-income and middle-income countries showed that anxiety disorders were associated with a substantial increase in the odds of suboptimal adherence (OR 1.59, 95\% CI 1.29 to 1.96). ${ }^{12}$ The strength of the association observed in our study was consistent with these meta-analyses.

Our findings show a substantial burden of CMD among people living with HIV in rural Zimbabwe and underline the need to integrate interventions for detecting and addressing CMD in this population. The Friendship Bench project is an evidence-based psychological intervention for delivering mental healthcare in primary care. ${ }^{21}$ The intervention has been rigorously evaluated in a large cluster randomised controlled trial in Harare, which showed that the Friendship Bench intervention substantially improved CMD symptoms. ${ }^{21}$ As part of the 


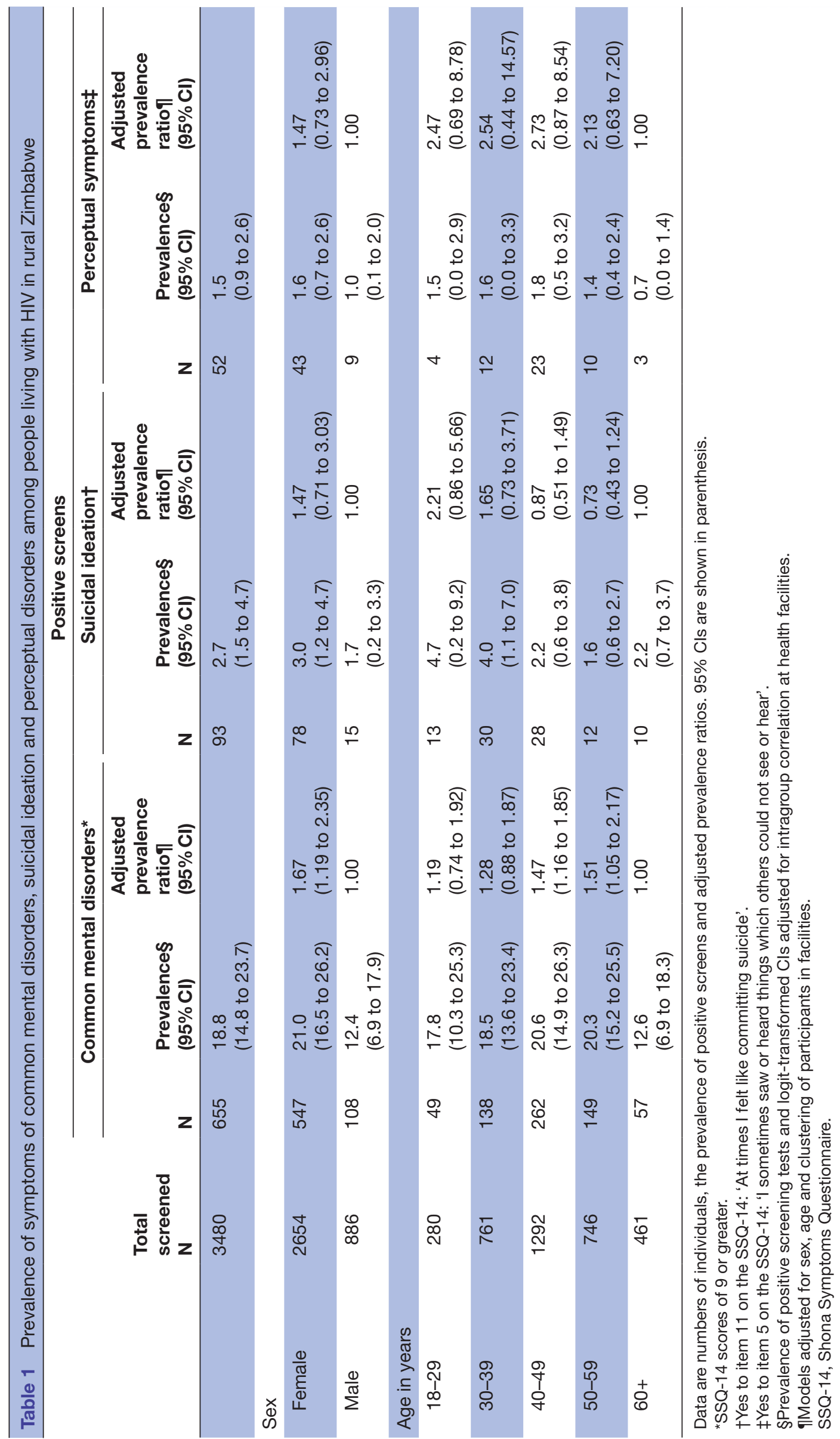


Table 2 Prevalence ratios for factors associated with suboptimal adherences among people living with HIV in rura Zimbabwe

\begin{tabular}{ll}
$\begin{array}{l}\text { Unadjusted } \\
\text { prevalence ratio } \\
(95 \% \mathrm{Cl})^{*}\end{array}$ & $\begin{array}{l}\text { Adjusted } \\
\text { prevalence ratio† } \\
(95 \% \mathrm{Cl})\end{array}$ \\
\hline
\end{tabular}

\begin{tabular}{lll}
\hline $\begin{array}{c}\text { CMD screening } \\
\text { Negative } \neq\end{array}$ & 1.00 & 1.00 \\
\hline $\begin{array}{l}\text { Positive§ } \\
\text { Sex }\end{array}$ & $1.46(1.29$ to 1.66$)$ & $1.53(1.37$ to 1.70$)$ \\
\hline $\begin{array}{l}\text { Female } \\
\text { Male }\end{array}$ & 1.00 & 1.00 \\
\hline Age in years & $1.17(0.96$ to 1.44$)$ & $1.25(1.01$ to 1.53$)$ \\
\hline $18-29$ & $1.60(1.10$ to 2.31$)$ & $1.62(1.10$ to 2.38$)$ \\
\hline $30-39$ & $1.29(0.90$ to 1.85$)$ & $1.31(0.91$ to 1.89$)$ \\
\hline $40-49$ & $1.00(0.72$ to 1.38$)$ & $0.99(0.71$ to 1.38$)$ \\
\hline $50-59$ & $1.04(0.72$ to 1.51$)$ & $1.03(0.71$ to 1.49$)$ \\
\hline $60+$ & 1.00 & 1.00 \\
\hline
\end{tabular}

Data are unadjusted and adjusted prevalence ratios. 95\% Cls are shown in parenthesis.

*Models adjusted for clustering of participants in facilities.

†Models adjusted for CMD screening, sex, age and clustering of participants in facilities.

$\ddagger S S Q-14$ scores of smaller than 9.

§SQ-14 scores of 9 or greater.

CMD, common mental disorders ; SSQ-14, Shona Symptoms

Questionnaire.

FB-ART trial, we have implemented the FB intervention at the eight intervention sites participating in the FB-ART trial, and will further roll out to the eight control sites to provide access to evidence-based mental health services for people attending these clinics.

Treatment of mental disorders among people living with HIV may also positively affect HIV treatment outcomes. ${ }^{1718}$ A meta-analysis of 29 observational studies and randomised trials showed that depression and psychological distress treatment enhances ART adherence. ${ }^{18}$ A further meta-analysis of three randomised controlled trials provides weak evidence for the benefit of cognitive behavioural therapy for depression and adherence on viral load suppression. ${ }^{17}{ }^{34-36}$ Despite these promising results, the evidence for the benefit of mental healthcare for improving HIV treatment outcomes is still limited.

Further work is needed to evaluate the effect of mental healthcare on the mental health of people living with HIV and HIV treatment outcomes. We are currently evaluating the effect of the Friendship Bench intervention on ART adherence, viral load suppression and symptoms of CMD among people living with HIV in rural Zimbabwe. There is also a need for continued routine programme monitoring and implementation science to ensure the quality and effectiveness of the Friendship Bench intervention in various settings.
In conclusion, our findings show a substantial burden of CMD among people living with HIV in rural Zimbabwe and underline the need to integrate mental health services in HIV treatment programmes.

Author affiliations

${ }^{1}$ Institute of Social \& Preventive Medicine (ISPM), University of Bern, Bern, Switzerland

${ }^{2}$ SolidarMed, Masvingo, Zimbabwe

${ }^{3}$ Friendship Bench Zimbabwe, Harare, Zimbabwe

${ }^{4}$ CTU Bern, University of Bern, Bern, Switzerland

${ }^{5}$ SolidarMed, Lucerne, Switzerland

${ }^{6}$ Department of Psychiatry, University of Zimbabwe, Harare, Zimbabwe

${ }^{7}$ Centre for Global Mental Health, London School of Hygiene and Tropical Medicine, London, UK

${ }^{8}$ Centre for Infectious Disease Epidemiology and Research, University of Cape Town, Cape Town, South Africa

${ }^{9}$ Population Health Sciences, Bristol Medical School, University of Bristol, Bristol, UK

Twitter Andreas D Haas @AndreasDHaas and Matthias Egger @eggersnsf Collaborators leDEA Southern Africa.

Contributors ME obtained funding for the study. ADH, JvD, ME, CK, DC, SH and AL wrote the study protocol. JM and RM assisted with fieldwork and data collection which was overseen by CK and JvD. SH and ADH did central data monitoring. ADH conducted statistical analysis. AL advised on statistical analysis. ADH drafted the initial manuscript. CK, SH, JM, JvD, RM, RV, AL, PMvG, EM, MH, DC and ME provided substantive edits to the manuscript. All authors contributed to interpretation of the results and have read and approved the final manuscript.

Funding The study was supported by the National Cancer Institute (NCl), the Eunice Kennedy Shriver National Institute of Child Health and Human Development (NICHD), the National Institute of Allergy and Infectious Diseases (NIAID), the National Institute of Mental Health (NIMH), the National Institute on Drug Abuse (NIDA), the National Heart, Lung and Blood Institute (NHLBI), the National Institute on Alcohol Abuse and Alcoholism (NIAAA), the National Institute of Diabetes and Digestive and Kidney Diseases (NIDDK) and the Fogarty International Centre (FIC) through the International epidemiology Databases to Evaluate AIDS (leDEA) (ME, grant number 5U01-Al069924-05) and the Swiss National Science Foundation (SNSF) (ME, grant number 189498 and AH, grant number 178602).

Disclaimer The funders had no role in study design, data collection and analysis, decision to publish or preparation of the manuscript.

Competing interests None declared.

Patient and public involvement Patients with psychiatric morbidity were involved in ethnographic and qualitative research, which informed the development of the SSQ-14 [24]. The results of this study were shared with the provincial medical director and the district medical officer.

Patient consent for publication Not required.

Ethics approval The authors assert that all procedures contributing to this work comply with the ethical standards of the relevant national and institutional committees on human experimentation and with the Helsinki Declaration of 1975 , as revised in 2008. The study protocol was approved by the ethics committees of the Medical Research Council of Zimbabwe (MRCZ) (approval number MRCZ/A/2287), the Research Council of Zimbabwe (RCZ), and the Canton of Bern, Switzerland (approval number 2018-00396). Individuals provided verbal consent for eligibility screening and collection and analysis of screening data. Eligible participants provided written informed consent to participate in the randomised controlled trial.

Provenance and peer review Not commissioned; externally peer reviewed.

Data availability statement Data are available on reasonable request. Data cannot be made available online because of legal and ethical restrictions. To request data, readers may contact leDEA for consideration by filling out the online form available at https://www.iedea-sa.org/contact-us/

Open access This is an open access article distributed in accordance with the Creative Commons Attribution Non Commercial (CC BY-NC 4.0) license, which permits others to distribute, remix, adapt, build upon this work non-commercially, and license their derivative works on different terms, provided the original work is 
properly cited, appropriate credit is given, any changes made indicated, and the use is non-commercial. See: http://creativecommons.org/licenses/by-nc/4.0/.

\section{ORCID iDs}

Andreas D Haas http://orcid.org/0000-0002-4849-181X

Ruth Verhey http://orcid.org/0000-0002-5959-1891

Matthias Egger http://orcid.org/0000-0001-7462-5132

\section{REFERENCES}

1 Joint United Nations Programme on HIV/AIDS (UNAIDS). AIDSInfo online database [Internet]. Available: http://www.aidsinfoonline.org/

2 Johnson LF, Mossong J, Dorrington RE, et al. Life expectancies of South African adults starting antiretroviral treatment: collaborative analysis of cohort studies. PLoS Med 2013;10:e1001418.

3 Haas AD, Zaniewski E, Anderegg N, et al. Retention and mortality on antiretroviral therapy in sub-Saharan Africa: collaborative analyses of HIV treatment programmes. J Int AIDS Soc 2018;21:e25084.

4 Ford N, Darder M, Spelman T, et al. Early adherence to antiretroviral medication as a predictor of long-term HIV virological suppression: five-year follow up of an observational cohort. PLoS One 2010;5:e10460.

5 Lofgren SM, Bond DJ, Nakasujja N, et al. Burden of depression in outpatient HIV-infected adults in sub-Saharan Africa; systematic review and meta-analysis. AIDS Behav 2020;24:1752-64.

6 Brandt C, Zvolensky MJ, Woods SP, et al. Anxiety symptoms and disorders among adults living with HIV and AIDS: a critical review and integrative synthesis of the empirical literature. Clin Psychol Rev 2017:51:164-84.

7 Kuehner C. Why is depression more common among women than among men? Lancet Psychiatry 2017;4:146-58.

8 Li SH, Graham BM. Why are women so vulnerable to anxiety, trauma-related and stress-related disorders? the potential role of sex hormones. Lancet Psychiatry 2017;4:73-82.

9 Chibanda D, Verhey R, Gibson LJ, et al. Validation of screening tools for depression and anxiety disorders in a primary care population with high HIV prevalence in Zimbabwe. J Affect Disord 2016;198:50-5.

10 Chibanda D, Cowan F, Gibson L, et al. Prevalence and correlates of probable common mental disorders in a population with high prevalence of HIV in Zimbabwe. BMC Psychiatry 2016;16:55.

11 Haas AD, Radin E, Hakim AJ, et al. Prevalence of nonsuppressed viral load and associated factors among HIV-positive adults receiving antiretroviral therapy in Eswatini, Lesotho, Malawi, Zambia and Zimbabwe (2015 to 2017): results from population-based nationally representative surveys. J Int AIDS Soc 2020;23.

12 Wykowski J, Kemp CG, Velloza J, et al. Associations between anxiety and adherence to antiretroviral medications in low- and middle-income countries: a systematic review and meta-analysis. AIDS Behav 2019;23:2059-71.

13 Haas AD, Technau K-G, Pahad S, et al. Mental health, substance use and viral suppression in adolescents receiving art at a paediatric HIV clinic in South Africa. J Int AIDS Soc 2020;23:e25644.

14 Haas AD, Ruffieux Y, van den Heuvel LL, Den HLLV, et al. Excess mortality associated with mental illness in people living with HIV in Cape town, South Africa: a cohort study using linked electronic health records. Lancet Glob Health 2020;8:e1326-34.

15 Uthman OA, Magidson JF, Safren SA, et al. Depression and adherence to antiretroviral therapy in low-, middle- and high-income countries: a systematic review and meta-analysis. Curr HIVIAIDS Rep 2014;11:291-307.

16 Meffert SM, Neylan TC, McCulloch CE, et al. East African HIV care: depression and HIV outcomes. Glob Ment Health 2019;6:e9.
17 Shi Y, Zhao M, Chen S, et al. Effects of cognitive behavioral therapy on people living with HIV and depression: a systematic review and meta-analysis. Psychol Health Med 2019;24:578-94.

18 Sin NL, DiMatteo MR. Depression treatment enhances adherence to antiretroviral therapy: a meta-analysis. Ann Behav Med 2014;47:259-69.

19 Ruffieux Y, Efthimiou O, Van den Heuvel LL, et al. The treatment gap for mental disorders in adults enrolled in HIV treatment programmes in South Africa: a cohort study using linked electronic health records. Epidemiol Psychiatr Sci 2021;30:e37.

20 Sikkema KJ, Dennis AC, Watt MH, et al. Improving mental health among people living with HIV: a review of intervention trials in lowand middle-income countries. Glob Ment Health 2015;2:1-26.

21 Chibanda D, Weiss HA, Verhey R, et al. Effect of a primary carebased psychological intervention on symptoms of common mental disorders in Zimbabwe: a randomized clinical trial. JAMA 2016;316:2618.

22 Chibanda D. Reducing the treatment gap for mental, neurological and substance use disorders in Africa: lessons from the Friendship bench in Zimbabwe. Epidemiol Psychiatr Sci 2017;26:342-7.

23 AIS. AIDS Indicator Survey (AIS) [Internet], 2017. Available: https:// dhsprogram.com/What-We-Do/Survey-Types/AIS.cfm

24 Patel V, Simunyu E, Gwanzura F, et al. The Shona symptom questionnaire: the development of an Indigenous measure of common mental disorders in Harare. Acta Psychiatr Scand 1997:95:469-75.

25 Cummings P. Methods for estimating adjusted risk ratios. Stata $J$ 2009;9:175-96.

26 Simoni JM, Kurth AE, Pearson CR, et al. Self-Report measures of antiretroviral therapy adherence: a review with recommendations for HIV research and clinical management. AIDS Behav 2006;10:227-45.

27 Seedat S, Scott KM, Angermeyer MC, et al. Cross-National associations between gender and mental disorders in the world Health organization world mental health surveys. Arch Gen Psychiatry 2009;66:785.

28 Kuehner C. Gender differences in unipolar depression: an update of epidemiological findings and possible explanations. Acta Psychiatr Scand 2003;108:163-74.

29 Oram S, Khalifeh H, Howard LM. Violence against women and mental health. Lancet Psychiatry 2017;4:159-70.

30 World Health Organization. Consolidated guidelines on the use of antiretroviral drugs for treating and preventing HIV infection recommendations for a public health approach. 2 edn. Geneva: World Health Organization, 2016.

31 leDEA and COHERE Cohort Collaborations. Global trends in CD4 cell count at the start of antiretroviral therapy: collaborative study of treatment programs. Clin Infect Dis 2018;66:893-903.

32 Ngum PA, Fon PN, Ngu RC, et al. Depression among HIV/AIDS patients on highly active antiretroviral therapy in the southwest regional hospitals of Cameroon: a cross-sectional study. Neurol Ther 2017:6:103-14

33 Office of the Surgeon General (US) Center for Mental Health Services (US), National Institute of Mental Health (US). Mental health: culture, race, and ethnicity: a supplement to mental health: a report of the surgeon General. Rockville, MD, 2001.

34 Safren SA, Bedoya CA, O'Cleirigh C, et al. Cognitive behavioural therapy for adherence and depression in patients with HIV: a threearm randomised controlled trial. Lancet HIV 2016;3:e529-38.

35 Safren SA, O'Cleirigh CM, Bullis JR, et al. Cognitive behavioral therapy for adherence and depression (CBT-AD) in HIV-infected injection drug users: a randomized controlled trial. J Consult Clin Psychol 2012;80:404-15.

36 Brown LK, Kennard BD, Emslie GJ, et al. Effective treatment of depressive disorders in medical clinics for adolescents and young adults living with HIV: a controlled trial. J Acquir Immune Defic Syndr 2016;71:38-46. 